\title{
Rhetorische Funktion von Projektorkonstruktionen mit deshalb und deswegen
}

\author{
Giorgio Antonioli (Innsbruck)
}

\begin{abstract}
This paper focuses on so called syntactic projection phenomena in the German language. This term from the German Gesprächsforschung is used to define the fact that an utterance or part of it foreshadows another one. This paper aims at pointing out how such projection phenomena are consciously exploited for rhethorical purposes. This will be observed on the basis of excerpts from the Stuttgart 21 mediation talks. The linguistic analysis carried out in this paper will focus on syntactic projection phenomena involving the use of causal adverbial connectives deshalb and deswegen.
\end{abstract}

\section{$1 \quad$ Einleitung}

Im vorliegenden Beitrag stelle ich einen Teil der Ergebnisse eines Postdoc-Projektes vor, das ich im Sommersemester 2016 an der Universität Duisburg-Essen durchgeführt habe. ${ }^{1}$ Das Projekt habe ich aus meiner Dissertation zum Thema Konnektoren im gesprochenen Deutsch (Antonioli 2016) herausentwickelt. Es beschäftigte sich mit dem Zusammenhang von Topologie, Syntax ${ }^{2}$, Prosodie und Informationsstruktur im mündlichen Gebrauch der sogenannten konnektintegrierbaren Konnektoren - eine ausführliche Erklärung dieses Begriffs erfolgt im Abschnitt 1.1. Diesen Zusammenhang habe ich versucht anhand einer empirischen Korpusanalyse nach Theorie und Methode der Interaktionalen Linguistik, d. h. mit Schwerpunkt auf (i) Form-Funktion-Paarung und (ii) Gattungsanalyse (vgl. Imo 2013: 77-78) nachzuvollziehen. Die Analyse hat gezeigt, wie bestimmte topologische und prosodische Muster in bestimmten Gattungen regelmäßig vorkommen, was m.E. ermöglicht, diesen Mustern gattungsspezifische interaktionale Funktionen zuzuschreiben. Im vorliegenden Beitrag werden Verwendungen der konnektintegrierbaren Kausalkonnektoren deshalb und deswegen gezeigt, die sich - in Anbetracht der gesamten untersuchten Datenmenge - als spezifisch für die kommunikative Gattung Schlichtungsgespräch erwiesen haben $-d$. h. für eine Gattung der öffentlichen Kommunikation, die sich durch persuasionsorientierte Handlungsziele auszeichnet. Dabei wird gezeigt, wie an Schlichtungsgesprächen beteiligte Redner solche Handlungsziele durch den Einsatz von rheto-

\footnotetext{
${ }^{1}$ An dieser Stelle möchte ich an Wolfgang Imo, Jens Lanwer, Eva Zitta, Georgios Coussios, Irina Mostovaia, Lisa Korte, Ying Ma und Bernhard Fisseni meinen tiefsten und herzlichsten Dank für ihre Hilfe und Unterstutzung während meines Aufenthalts aussprechen.

${ }^{2}$ Im Anliegen des vorliegenden Beitrages bezieht sich der Begriff Topologie auf die vom Konnektor besetzte Position in der Äußerung, während sich der Begriff Syntax auf die gesamte Struktur der Äußerung bezieht.
} 
rischen Hilfsmitteln verfolgen, indem sie die Projektionskraft bestimmter Sprachstrukturen ausnutzen, und wie sich die oben genannten Konnektoren deshalb und deswegen dabei als Auslöser von Projektorkonstruktionen erweisen.

Die Arbeit gliedert sich folgendermaßen. In den folgenden Abschnitten werden ausführlichere Erklärungen zu den oben erwähnten Begriffen geliefert, nämlich konnektintegrierbaren Konnektoren (1.1.) und Projektorkonstruktionen (1.2.). Anschließend wird eine Übersicht über Daten und Methode gegeben (2). Die Ergebnisse der empirischen Analyse werden in (3) dargestellt und ausgewertet und im abschließenden Fazit (4) zusammengefasst.

\subsection{Konnektintegrierbare Konnektoren}

Der Begriff ist dem ersten Handbuch der deutschen Konnektoren (Pasch et al. 2003, im Folgenden HdK1) entliehen und bezeichnet diejenigen Konnektoren, die in einem ihrer beiden Argumente - im Folgenden Konnekte - syntaktisch integriert werden können. Wie dies erfolgt, wird unten am Beispiel von aber veranschaulicht - vgl. dazu HdK1 (6-12):

\begin{tabular}{|c|c|c|}
\hline Die Sonne scheint, & aber & sie wollen nicht wandern gehen \\
\hline EXTERNES KONNEKT & NICHT KONNEKTINTEGRIERTER KONNEKTOR & INTERNES KONNEKT \\
\hline
\end{tabular}

Abbildung 1: Nicht konnektintegriertes aber

In Abb.1 erscheint aber zwischen seinen beiden Konnekten - nach der Terminologie des Handbuchs besetzt es die Nullposition. In konnektintegrierter Position besetzt aber das Mittelfeld:

\begin{tabular}{c|c}
\hline Die Sonne scheint, & sie wollen aber nicht wandern gehen \\
\hline EXTERNES KONNEKT & INTERNES KONNEKT MIT KONNEKTINTEGRIERTEM KONNEKTOR
\end{tabular}

Abbildung 2: Konnektintegriertes aber

Die im vorliegenden Beitrag untersuchten Konnektoren, deshalb und deswegen, gehören ebenso zur syntaktischen Makroklasse der konnektintegrierbaren Konnektoren. Ihre topologischen Eigenschaften lassen sich anhand der HdK-Terminologie folgendermaßen beschreiben:

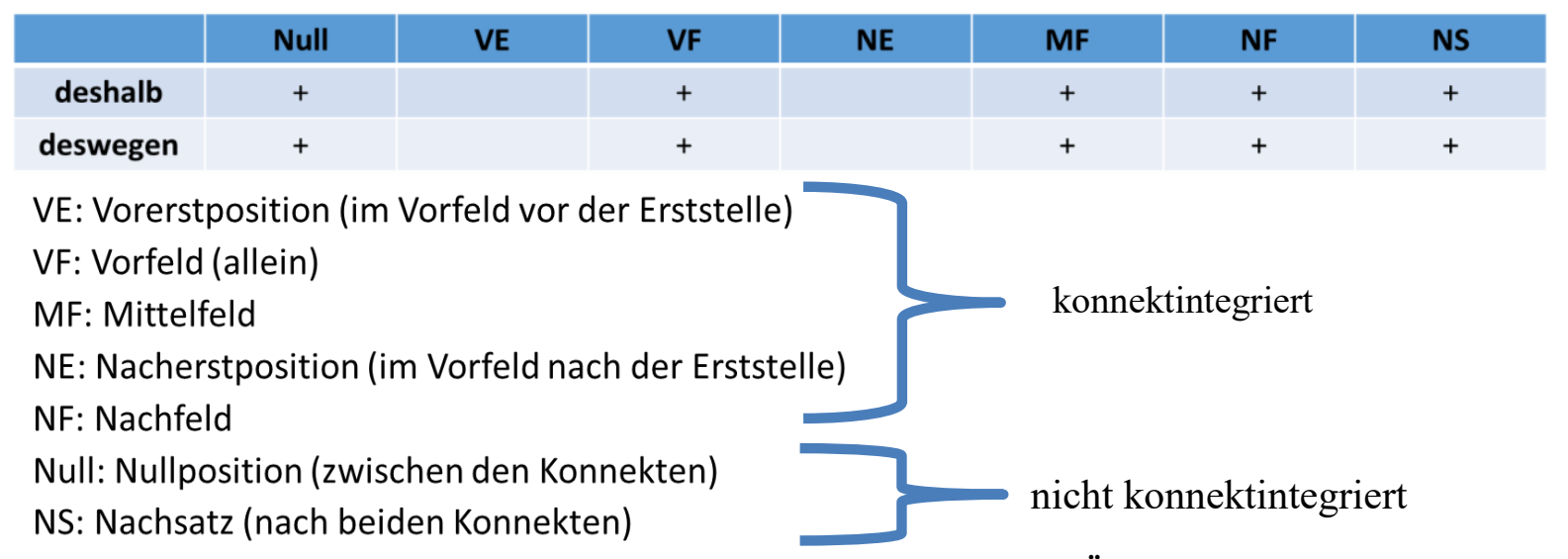

Abbildung 3: deshalb und deswegen - topologische Übersicht

Für deshalb und deswegen liegen also drei konnektintegrierte und zwei nicht konnektintegrierte Verwendungen vor.

Die nicht konnektintegrierten Verwendungen sind in der Nullposition und in der Nachsatzposition: 
Nullposition: Der Autor ist krank geworden. Deswegen: Die Lesung wird auf den Januar 2001 verschoben.

Nachsatz ${ }^{3}$ : Die Lesung wird auf den Januar 2001 verschoben. Der Autor ist krank geworden. Deswegen.

(HdK1: 495-497, Hervorhebungen im Original)

Bei den konnektintegrierten Verwendungen stehen deshalb und deswegen alleine im Vorfeld ${ }^{4}$, im Mittelfeld und im Nachfeld:

Vorfeld: Der Autor ist krank geworden. Deswegen wird die Lesung auf den Januar verschoben. Mittelfeld: Der Autor ist krank geworden. Die Lesung wurde deswegen auf den Januar verschoben.

Nachfeld: Der Autor ist krank geworden. Die Lesung wurde auf den Januar verschoben deswegen.

(HdK1: 495-497, Hervorhebungen im Original)

Für die konnektintegrierten deshalb und deswegen liegt eine zusätzliche Verwendung vor, die das eigentliche Thema des vorliegenden Beitrages ist, und zwar die in Korrelation mit dem Subjunktor weil. Diese wird im zweiten Handbuch der deutschen Konnektoren (Breindl/Volodina/Waßner 2014, im Folgenden HdK2) durch die folgenden Beispiele veranschaulicht:

Wer genaue Details hören wollte, wurde auf der anschließenden Pressekonferenz enttäuscht. „Wir haben Vertraulichkeit vereinbart“, erklärte Hermann. Dies sei auch deswegen notwendig, weil die Bahn bei der Vergabe von Aufträgen für Stuttgart 21 eine Wettbewerbsverzerrung vermeiden wolle, so Kefer. (Mannheimer Morgen, 22.01.2013, S. 8)

Manche Gegenden könnten mit bis zu 30 Zentimetern Regen durchtränkt, andere mit bis zu 60 Zentimetern Schnee bedeckt werden. Der Sturm ist deshalb so gefährlich und ungewöhnlich, weil er erst ganz zum Ende der Hurrikansaison und zum Beginn der Winterstürme zuschlägt. (Hamburger Morgenpost, 29.10.2012, S. S02)

(HdK2: 891, Hervorhebungen im Original)

Anhand dieser beiden Beispiele für deshalb/deswegen-weil-Korrelationen lässt sich beobachten, wie das jeweils konnektintegrierte deshalb-deswegen den darauffolgenden weil-Nebensatz de facto antizipiert und für den Rezipienten - in diesem Fall für den Leser - erwartbar macht. Auf diese Art der Antizipierung wird im Folgenden durch den Begriff syntaktische Projektion Bezug genommen. Dieser wird im folgenden Abschnitt ausfürhlich erläutert.

\subsection{Syntaktische Projektion und Projektorkonstruktionen}

Der Begriff der syntaktischen Projektion geht auf Auer (2002:1) zurück und bezeichnet „the fact that an individual action of part of it foreshadows another one“ - also die Tatsache, dass

\footnotetext{
${ }^{3}$ Die Nachsatzposition unterscheidet sich darin von der Nachfeldposition, dass der Konnektor im Nachsatz als selbständige syntaktische Einheit erscheint. Diese Selbständigkeit wird im HdK1-Beispiel mithilfe der Interpunktion dargestellt - deswegen ist nämlich durch den Punkt von seinem zweiten Konnekt getrennt.

${ }^{4}$ Das HdK1 (494-500) unterscheidet drei Arten der Vorfeldbesetzung. Neben der Alleinbesetzung des Vorfelds nennt es auch (i) die Besetzung der Vorerstposition, in der der Konnektor vor einer zusätzlichen Vorfeldkonstituente erscheint (Sie fordern bessere Lebensbedingungen. Nur das setzt eine alternative Politik voraus) bzw. (ii) die Besetzung der Nacherstposition, in der der Konnektor nach einer zusätzlichen Vorfeldkonstituente erscheint (Sie fordern bessere Lebensbedingungen. Das allerdings setzt eine alternative Politik voraus). Nacherst- und Vorerstposition können deshalb und deswegen nicht besetzen.
} 
eine individuelle Handlung oder Teilhandlung eine andere voraussichtbar macht. Den Begriff führt Auer im Rahmen seiner Theorie der inkrementellen Syntax ein, die gesprochene Sprache als prozesshaftes und zeitliches Phänomen versteht (siehe auch Auer 2007: 96-97). Er geht davon aus, dass die gesprochene Sprache (i) linear in der Zeit, (ii) kooperativ und (iii) auf kognitiver Basis produziert wird und spricht syntaktischen Projektionen in der jeweiligen Hinsicht eine wesentliche Rolle zu. Diese begünstigen die reibungslose Produktion und Rezeption von Redebeiträgen in der Echtzeit, indem sie durch Rückgriff auf kognitiv verfestigte Sprachmuster durch den Sprecher dem Hörer die Vorhersage von möglichen Redezug-Abschlusspunkten ermöglichen. Mit der Erforschung von derartigen verfestigten projektionsfähigen Sprachmustern oder Projektorkonstruktionen hat sich v.a. die Interaktionale Linguistik beschäftigt. Nennenswert sind u. a. die Arbeiten von Susanne Günthner $(2006,2008)$ zu zweigliedrigen Konstruktionen wie:

(i) Pseudocleft-Konstruktionen:

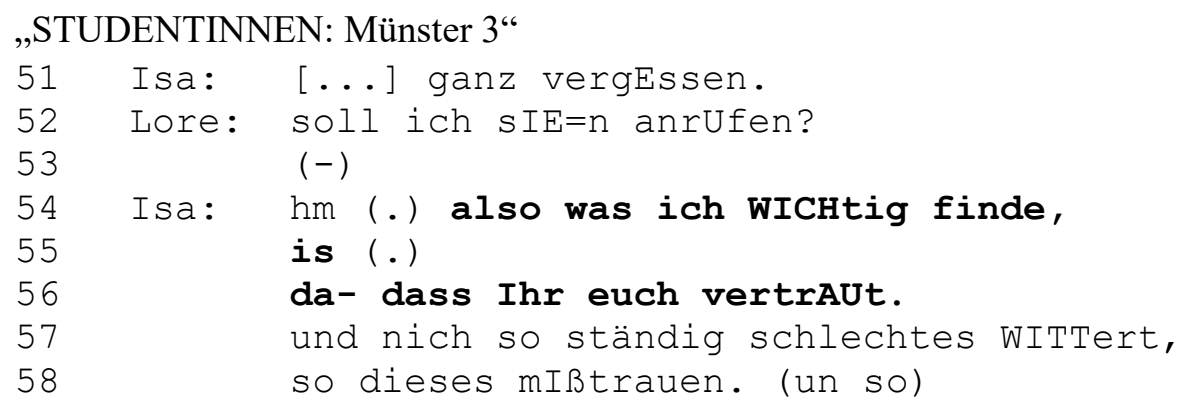

(Günthner 2008: 92)

(ii) $\quad$ die Sache ist $]$-Konstruktionen:

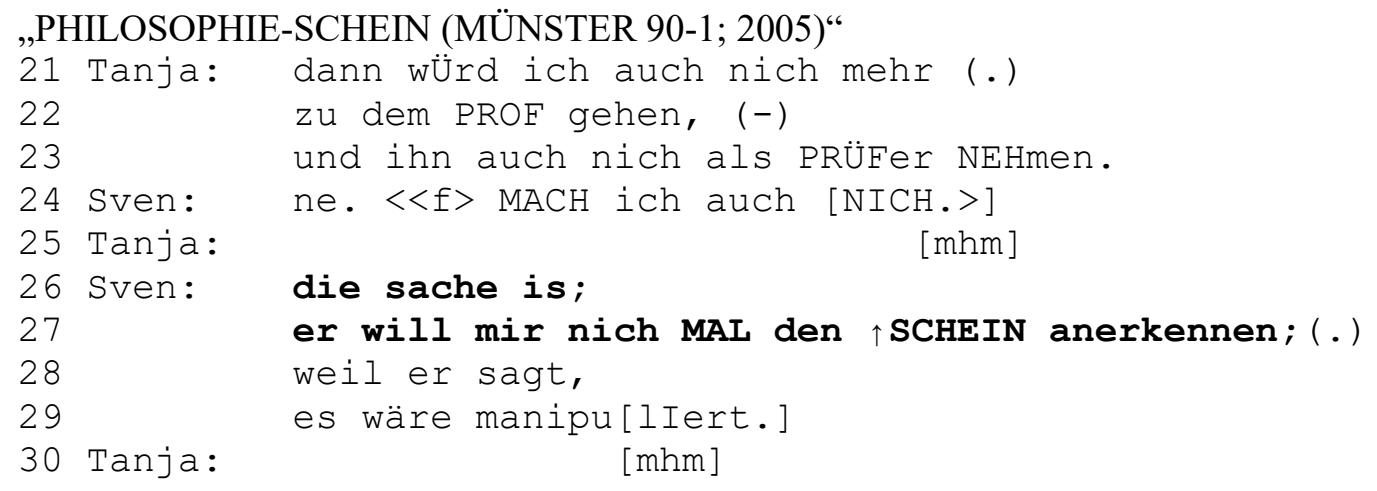

(Günthner 2008: 99-100)

Günthner hat gezeigt, wie derartige Projektorkonstruktionen dem Sprecher die zeitlich lineare Gestaltung von komplexen syntaktischen Strukturen und die Präsentierung von komplexen Sachverhalten ermöglichen, indem sie nämlich den Hörer von der Übernahme des Rederechts abhalten. Das erfolgt dadurch, dass das erste Glied der jeweiligen Konstruktion - was-Satz in (i) und [die Sache ist] in (ii) - eine Spannung erzeugt, die die Aufmerksamkeit des Hörers auf das noch zu artikulierende zweite Glied lenkt. Zweigliedrige Projektorkonstruktionen gelten also nicht nur als interaktionale Ressource für den syntaktischen Aufbau der Kommunikation, sondern auch für die pragmatische Relevanzstufung und somit für die Gestaltung der Informationsstruktur. Genau das erfolgt in den [deshalb/deswegen, weil]-Korrelationen in 1.1., denn die Verwendung von deshalb/deswegen gibt dem Rezipienten zu verstehen, dass die im ersten Glied geäußerte Proposition im zweiten Glied anschließend begründet werden soll. 
Im nächsten Abschnitt wird auf den Gebrauch von [deshalb/deswegen, weil]-Projektorkonstruktionen im gesprochenen Deutsch näher eingegangen, indem eine Übersicht über die untersuchten Daten und über die Methode zu ihrer Auswertung gegeben wird.

\section{Daten und Methode}

Für meine empirische Analyse habe ich eine Stichprobe aus dem FOLK-Korpus der Datenbank des gesprochenen Deutsch (DGD) des Instituts für deutsche Sprache in Mannheim ausgewählt. Die untersuchten Projektorkonstruktionen erscheinen in verschiedenen Gattungen der privaten, der öffentlichen und der institutionellen Kommunikation, die in der folgenden Tabelle gezeigt werden - die Benennungen der einzelnen Gattungen entsprechen der DGD-Terminologie:

\begin{tabular}{|l|l|l|l|l|l|}
\hline $\begin{array}{l}\text { INSTITUTIONELLE } \\
\text { KOMMUNIKATION }\end{array}$ & deshalb & deswegen & ALLTAGSKOMMUNIKATION & deshalb & deswegen \\
\hline Schlichtungsgespräch & $\mathbf{1 0}$ & $\mathbf{1 6}$ & Tischgespräch & $\mathbf{3}$ & $\mathbf{2}$ \\
\hline Fahrschulgespräch & $\mathbf{1}$ & $\mathbf{3}$ & $\begin{array}{l}\text { Spielinteraktion zwischen } \\
\text { Erwachsenen }\end{array}$ & $\mathbf{2}$ & $\mathbf{1}$ \\
\hline Prüfungsgespräch & $\mathbf{5}$ & $\mathbf{5}$ & Gespräch beim Renovieren & $\mathbf{2}$ \\
\hline $\begin{array}{l}\text { Meeting bei sozialer } \\
\text { Einrichtung }\end{array}$ & $\mathbf{6}$ & Kommunikation beim Kochen & $\mathbf{2}$ & $\mathbf{2}$ \\
\hline Interview & $\mathbf{2}$ & $\mathbf{2}$ & Sonstige & $\mathbf{1}$ \\
\hline Sonstige & $\mathbf{1 8}$ & $\mathbf{4 0}$ & & $\mathbf{4}$ \\
\hline Gesamt & & & & $\mathbf{8}$ \\
\hline
\end{tabular}

Tabelle 1: Distribution und Häufigkeit von [deshalb/deswegen, weil]-Projektorkonstruktionen im FOLK

Wie an der Tabelle abzulesen ist, erscheint die untersuchte Konstruktion am häufigsten in der Gattung Schlichtungsgespräch, was m.E. auf die in 1 genannten gattungsspezifischen Handlungsziele zurückzuführen ist:

„Das Handlungsziel von Schlichtung besteht darin, zwischen zwei Streitenden eine Regelung zustande zu bringen, die beide akzeptieren können. Dazu bedarf es idealiter eines Einstellungswandels bei den Streitparteien. Gleichzeitig verfolgen die Parteien ihre individuellen Handlungsziele, die sie nicht gegen und besser mit dem Schlichter durchsetzen können. So erfolgen die Aktivitäten aller drei Beteiligten in der Absicht, den oder die je anderen für das eigene Handlungsziel zu beeinflussen. Diese Aktivitäten sind persuasiver Natur."

(Nothdurft 1995: 20)

Die gegenseitige Beeinflussung erfolgt durch die Anführung von Argumenten zur Begründung der eigenen Einstellung. In den Gesprächen aus dem Korpus, die aus dem Schlichtungsverfahren zum „Stuttgart 21“-Projekt stammen, bestehen die Argumente in „Sachen und Fakten“, wie der Schlichter Heiner Geißler in seinem Grußwort auf der offiziellen Webseite zur Schlichtung betont: 
Das Schlichtungsverfahren war eine sogenannte Sach- und Faktenschlichtung. Sie sollte und soll weiter dazu dienen, Sie über Zahlen, Daten und Fakten rund um Stuttgart 21 zu informieren, damit Sie sich ein eigenes Urteil bilden können.

(www.schlichtung-s21.de/ [23.06.19])

Der Gebrauch der untersuchten Konstruktion in der genannten Gattung unterscheidet sich von den übrigen Korpusbelegen nicht nur in der Häufigkeit, sondern auch in der Art. Für die Realisierung von [deshalb/deswegen, weil]-Konstruktionen liegen in den untersuchten Schlichtungsgesprächen zwei wiederkehrende Muster vor, die in den anderen Gattungen nicht nachgewiesen werden konnten:

1. deshalb am rechten Rand

2. [Nominalphrase + deswegen]-Matrixphrase

Ihre kennzeichnenden formalen Merkmale liegen dabei in den topologisch-syntaktischen ${ }^{5}$ und prosodischen Eigenschaften des konnektintegrierten deshalb/deswegen. Genau von diesen formalen Eigenschaften geht die Analyse aus und setzt sich zum Ziel, nachzuweisen, wie sich diese auf die Informationsstruktur niederschlagen.

Für die Beschreibung auf informationsstruktureller Ebene wird auf die folgenden Kategorien zurückgegriffen:

- Relativ präsupponierende Äußerungseinheiten, d. h. Äußerungseinheiten, deren propositionaler Gehalt dem Hörer vertraut ist, bzw.

- Relativ assertierende Äußerungseinheiten, d. h. Äußerungseinheiten, deren propositionaler Gehalt dem Hörer nicht vertraut ist

Diese beiden Kategorien, die von Auer (1998) für die Analyse von [Matrixsatz + Abhängiger Hauptsatz]-Konstruktionen übernommen wurden, sind für die informationsstrukturelle Analyse von zweigliedrigen syntaktischen Konstruktionen m.E. besonders geeignet, denn sie ermöglichen, das informationsstrukturelle Gewicht der einzelnen Glieder und somit ihre pragmatische Relevanzhochstufung bzw. -rückstufung zu bestimmen. ${ }^{6}$

\section{Datenanalyse}

Im Folgenden werden die in 2 genannten wiederkehrenden Muster im Gebrauch der [deshalb/deswegen, weil]-Projektorkonstruktion in den untersuchten Korpusdaten ausführlich be-

\footnotetext{
${ }^{5}$ Unter topologisch-syntaktischen Eigenschaften werden im Anliegen des vorliegenden Beitrages die Eigenschaften verstanden, die sich auf die vom Konnektor besetzte Position in der Bezugsäußerung im engeren Sinne (Topologie) bzw. auf die Struktur der Bezugsäußerung im weiteren Sinne (Syntax) beziehen.

${ }^{6}$ Sowohl die Kategorien für die informationsstrukturelle Analyse als auch die Terminologie für die topologische und syntaktische Beschreibung von deshalb/deswegen wurden mir von Wolfgang Imo im Laufe von Einzelbesprechungen während meines Aufenthaltes an der Universität Duisburg-Essen vorgeschlagen. Die Terminologie wird im folgenden Abschnitt im Einzelnen begründet.
} 
schrieben. Die vorgestellten Sprachdaten bestehen aus Transkripten nach den GAT2-Konventionen $^{7}$ (vgl. Selting et al. 2009) und aus den graphischen Darstellungen der entsprechenden Intonationsverläufe durch PRAAT.

\section{1. deshalb am rechten Rand}

Unter Besetzung des rechten Rands wird an dieser Stelle das Erscheinen des konnektintegrierten deshalb im rechten Mittelfeld und im Nachfeld verstanden. Die Wahl eines Oberbegriffs erfolgte in Anbetracht der Tatsache, dass sich die Topologie von deshalb/deswegen bei der Auswertung der Daten an manchen Stellen als mehrdeutig erwies. Das betrifft Äußerungen mit offener Klammerstruktur, in denen die rechte Satzklammer nicht festgestellt werden kann und die Abgrenzung von Mittelfeld und Nachfeld dementsprechend unscharf bleibt.

Als erstes Beispiel wird eine Verwendung von deshalb im rechten Mittelfeld angeführt, d. h. in einer satzwertigen Äußerung mit offener Klammerstruktur:

Beispiel (1), Sprechereignis FOLK_00064; DGD-Kennzeichnung: Schlichtungsgespräch

Redner: Brigitte Dahlbender (Gegnerin)

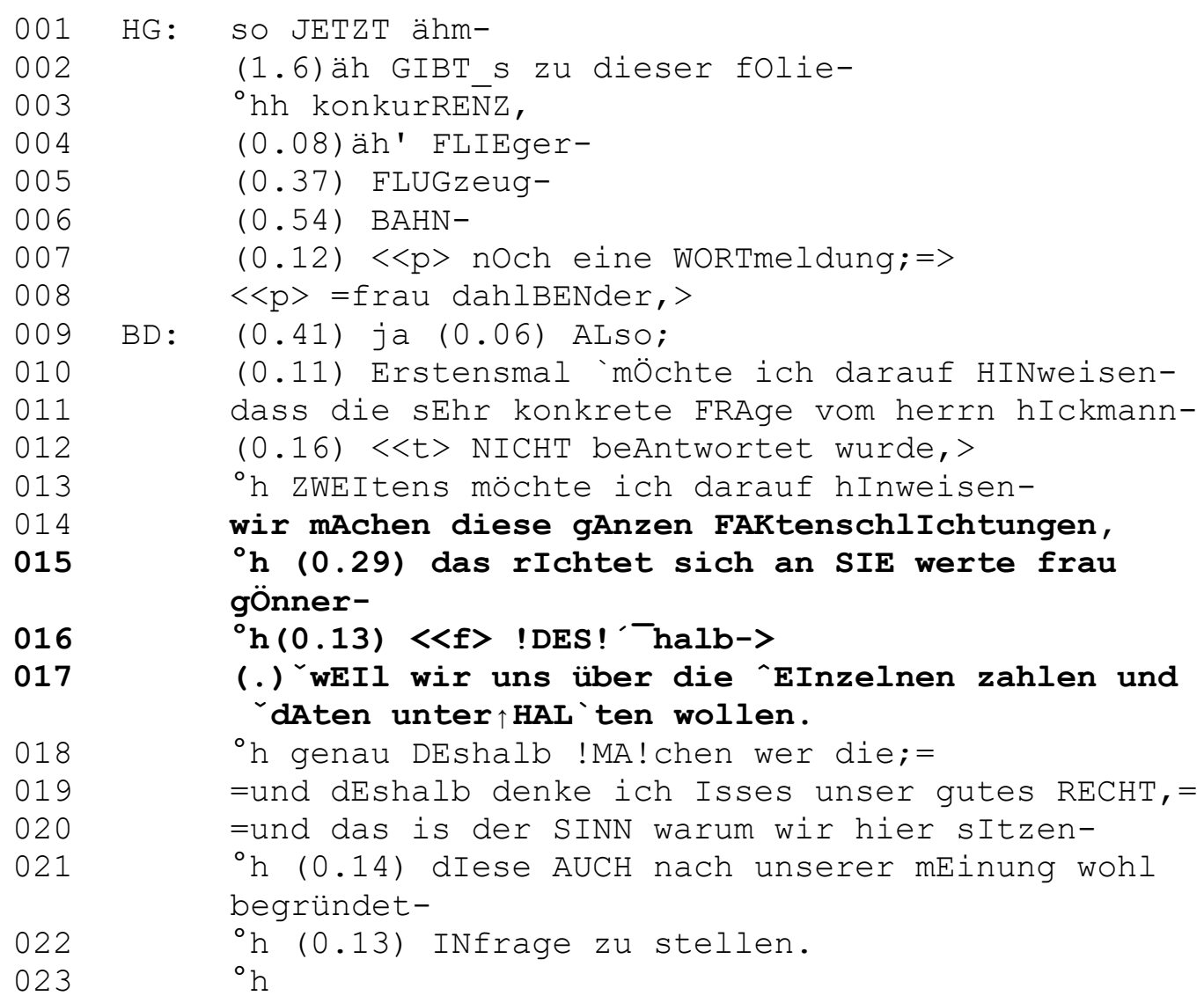

Die Rednerin produziert einen langen und komplexen listenartigen Redebeitrag (Erstensmal, 010; ZWEItens, 013). An der markierten Stelle schiebt sie eine Anrede parenthetisch ein (das rIchtet sich an SIE werte frau gÖnner-, 015), die das Mittelfeld aufspaltet. Anschließend

\footnotetext{
${ }^{7}$ Die für die Untersuchung relevanten Stellen sind fett markiert und in Feintranskript wiedergegeben, ihr Umfeld in Basistranskript.
} 
kommt sie auf den Leitfaden ihres Arguments zurück, und zwar durch die Markierung des prosodisch selbständigen !DES!'- halb (016), das mit starkem Akzent und erhöhter Lautstärke betont wird. Zugleich bringt sie Spannung ein, indem sie ihrer Hörerschaft zu verstehen gibt, dass ihr Redebeitrag noch nicht abgeschlossen ist. Das wird durch die gleichbleibende Tonkontur markiert:

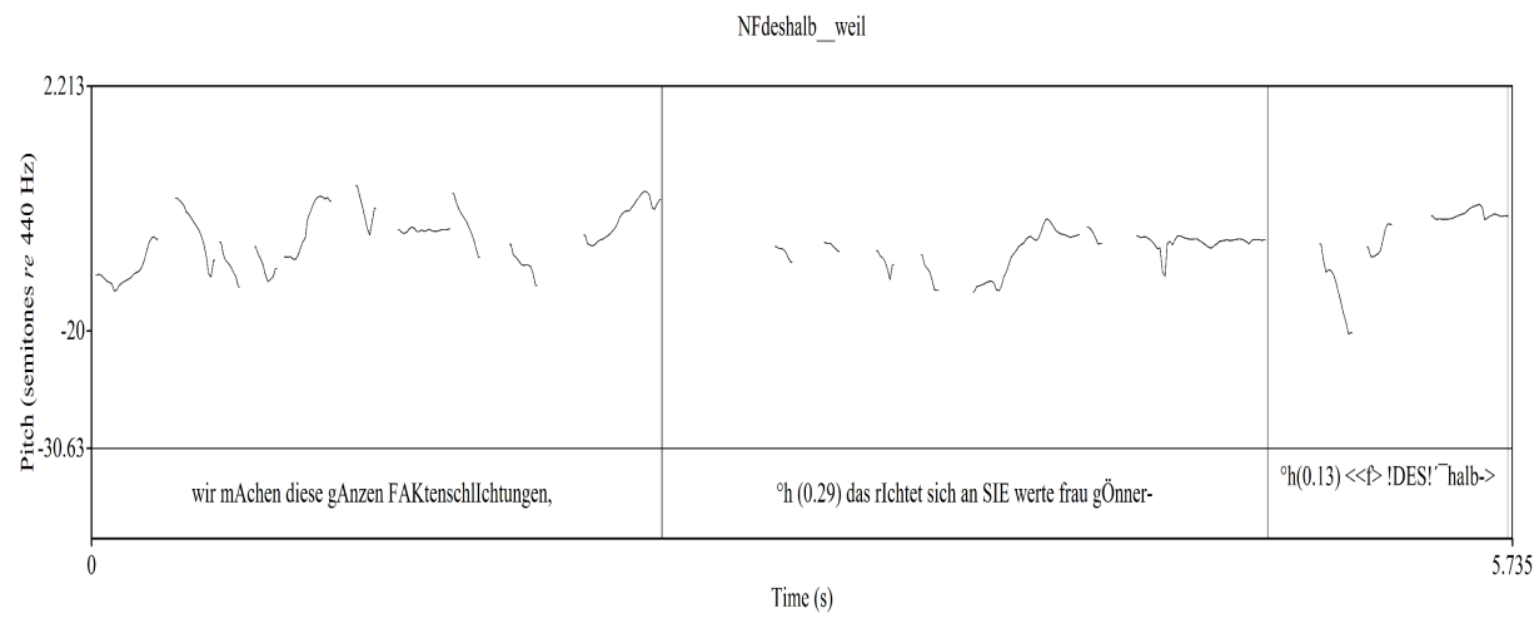

Abbildung 4: Beispiel (1), Tonhöhenverlauf 014-016

Insofern, als die gleichbleibende Tonkontur als Hinweis auf semantische Unabgeschlossenheit gedeutet wird, kann m.E. behauptet werden, dass die Rednerin dadurch Erwartung an eine Begründung schafft, die geliefert werden soll. Demzufolge lenkt sie die Aufmerksamkeit ihrer Hörerschaft auf das noch zu artikulierende zweite Glied der Projektorkonstruktion, d. h. auf den weil-Satz (017). Ein weiteres wichtiges prosodisches Merkmal dieser Verwendung von deshalb liegt darin, dass dieses einen starken Akzent trägt. In Anbetracht der Tatsache, dass die Vorgängerstruktur von deshalb ebenfalls einen starken Akzent trägt (FAKtenschlIchtungen, 014), ergibt sich ein Satz mit bifokaler Informationsstruktur (FAKtenschlIchtungen, !DES!' halb). Dadurch werden die beiden syntaktischen Glieder der Projektorkonstruktion als relativ assertierende Informationseinheiten markiert, d. h. sie werden hinsichtlich ihrer Relevanz pragmatisch gleichermaßen hochgestuft. Die Rednerin macht durch die starke Akzentuierung von FAKtenschlIchtungen ihre Hörerin darauf aufmerksam, dass in der laufenden Schlichtung Fakten zur Debatte stehen. Demzufolge kann ihre Äußerung als Aufforderung zur Sachlichkeit verstanden werden. Diese Aufforderung emphatisiert sie zusätzlich durch die prosodische Abgrenzung und starke Akzentuierung von !DES!' halb, die die Relevanzhochstufung auf dessen

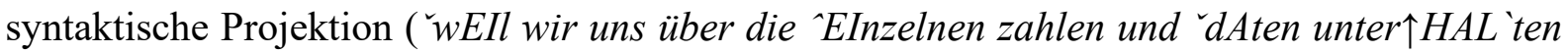
wollen, 017) erweitert.

Als nächstes Beispiel für die Besetzung des rechten Rands wird nun eine Verwendung von deshalb im Nachfeld gezeigt:

Beispiel (2): Sprechereignis FOLK_00069; DGD-Kennzeichnung: Schlichtungsgespräch

Redner: Prof. Walter Lächler (Sachverständiger)

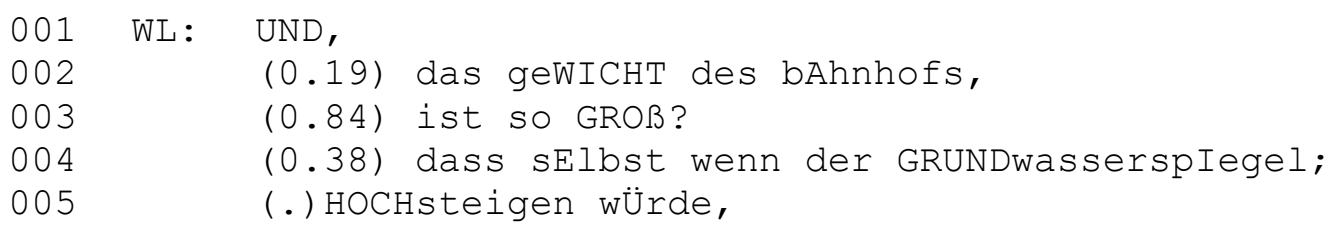




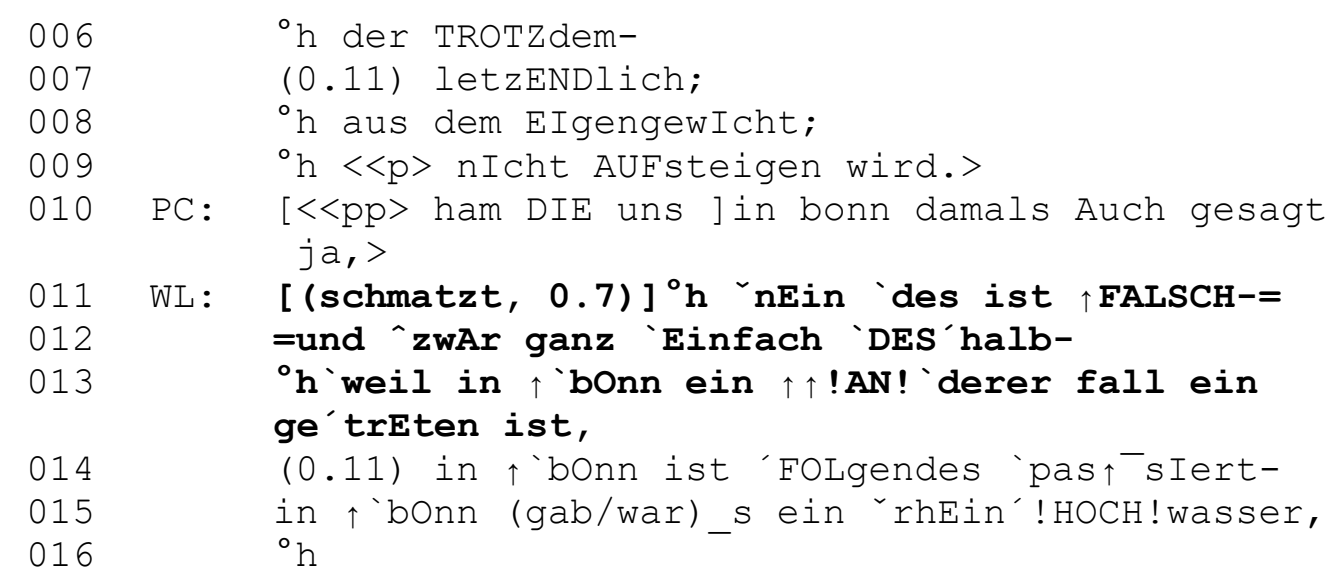

Der Redner reagiert an der fett markierten Stelle auf einen Einwand. Als Sachverständiger argumentiert er dafür, dass der Bau des Stuttgart-21-Bahnhofs den Grundwasserspiegel unberührt lässt (001-009). Eine der Parteien wendet ein, ein solches Argument sei in einem analogen Vorfall in Bonn bereits angeführt worden - und habe sich dann als falsch erwiesen (ham DIE uns in bonn damals Auch gesagt, 010). Um den Einwand zu erwidern, greift der Redner auf eine bifokale Informationsstruktur zurück. In erster Linie lehnt er den Einwand ab ( ${ }^{2} n E i n ` d e s$ ist $\uparrow F A L S C H-$, 011) und anschließend kündigt er die Angabe seiner Begründung vor (=und 'zwAr ganz 'Einfach 'DES'halb-, 012). Das konnektintegrierte deshalb erscheint immer noch am rechten Rand des Hauptsatzes, diesmal aber im Nachfeld - die Einleitungsfloskel und zwar weist darauf hin, dass es sich um einen Nachtrag handelt (vgl. Altmann 1981: 70-72). Auf prosodischer Ebene weist diese Projektorkonstruktion jedoch die gleichen Eigenschaften wie in Beispiel (1) auf, nämlich starke Akzentuierung von deshalb, prosodische Abgrenzung von seiner Vorgängerstruktur und gleichbleibende Tongrenze:

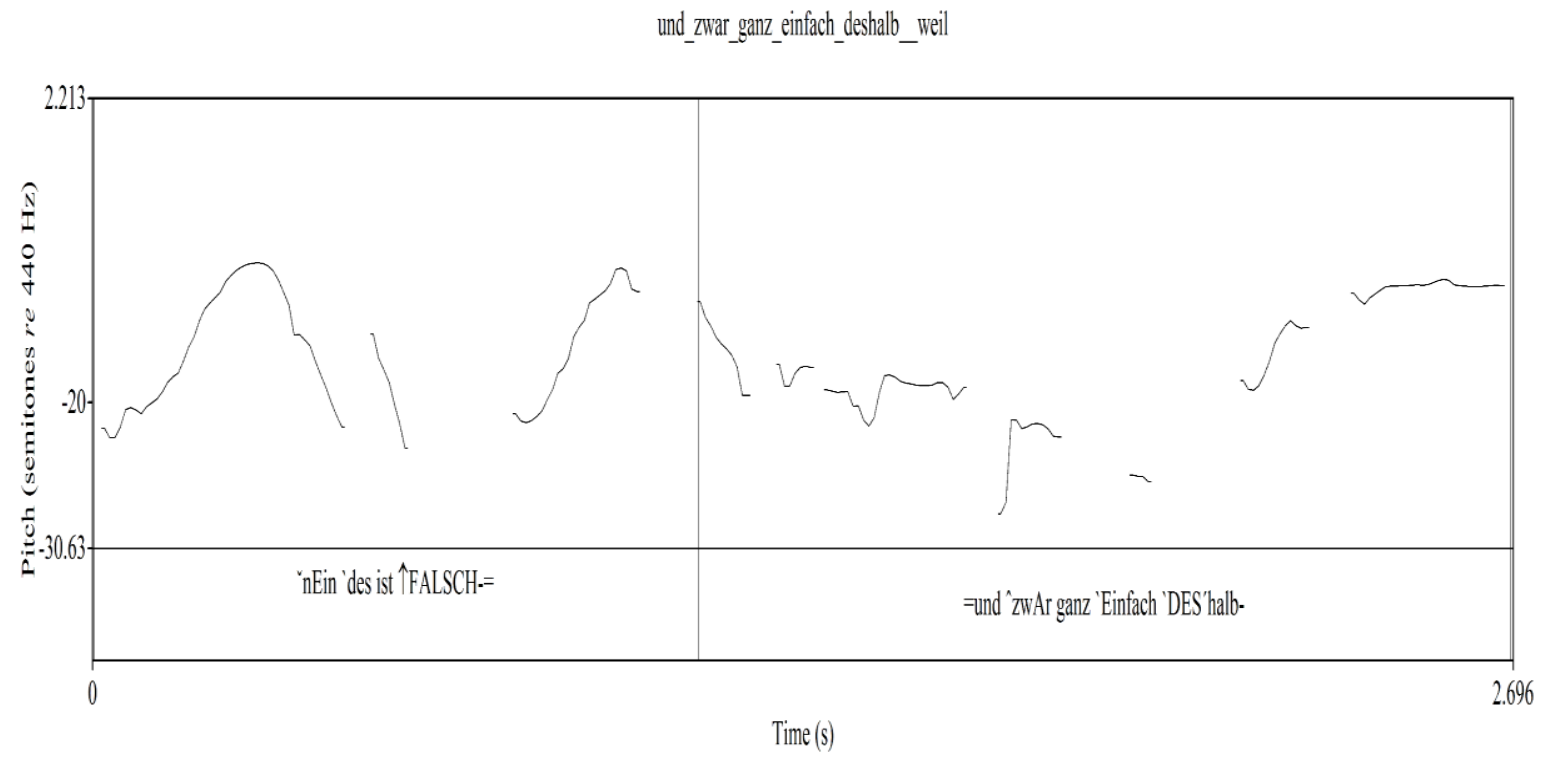

Abbildung 5: Beispiel (2), Tonhöhenverlauf 011-012

Die zwei Glieder dieser Projektorkonstruktion bringen also zwei distinkte Sprechhandlungen zum Ausdruck. Im ersten Teil wird eine Ablehnung formuliert, im zweiten Teil ihre Begründung. Der Übergang von der ersten zur zweiten erfolgt nicht direkt, sondern der Nachtrag dient als Überbrückung. Der Redner bringt also eine relativ assertierende komplexe Äußerung ins 
Gespräch ein und portioniert ihren Informationsgehalt in kleinere Einheiten, damit diese als gleichermaßen hochrelevant rezipiert werden und die Rede nicht an Spannung verliert.

\section{2 [Nominalphrase + deswegen]-Matrixphrase}

Im zweiten wiederkehrenden Muster erscheint der Konnektor in einer nicht satzwertigen Äußerungseinheit, er ist nämlich einer Nominalphrase - im Folgenden NP - nachgestellt:

Beispiel (3): Sprechereignis FOLK_00070; DGD-Kennzeichnung: Schlichtungsgespräch

Redner: Volker Kefer (Befürwörter)

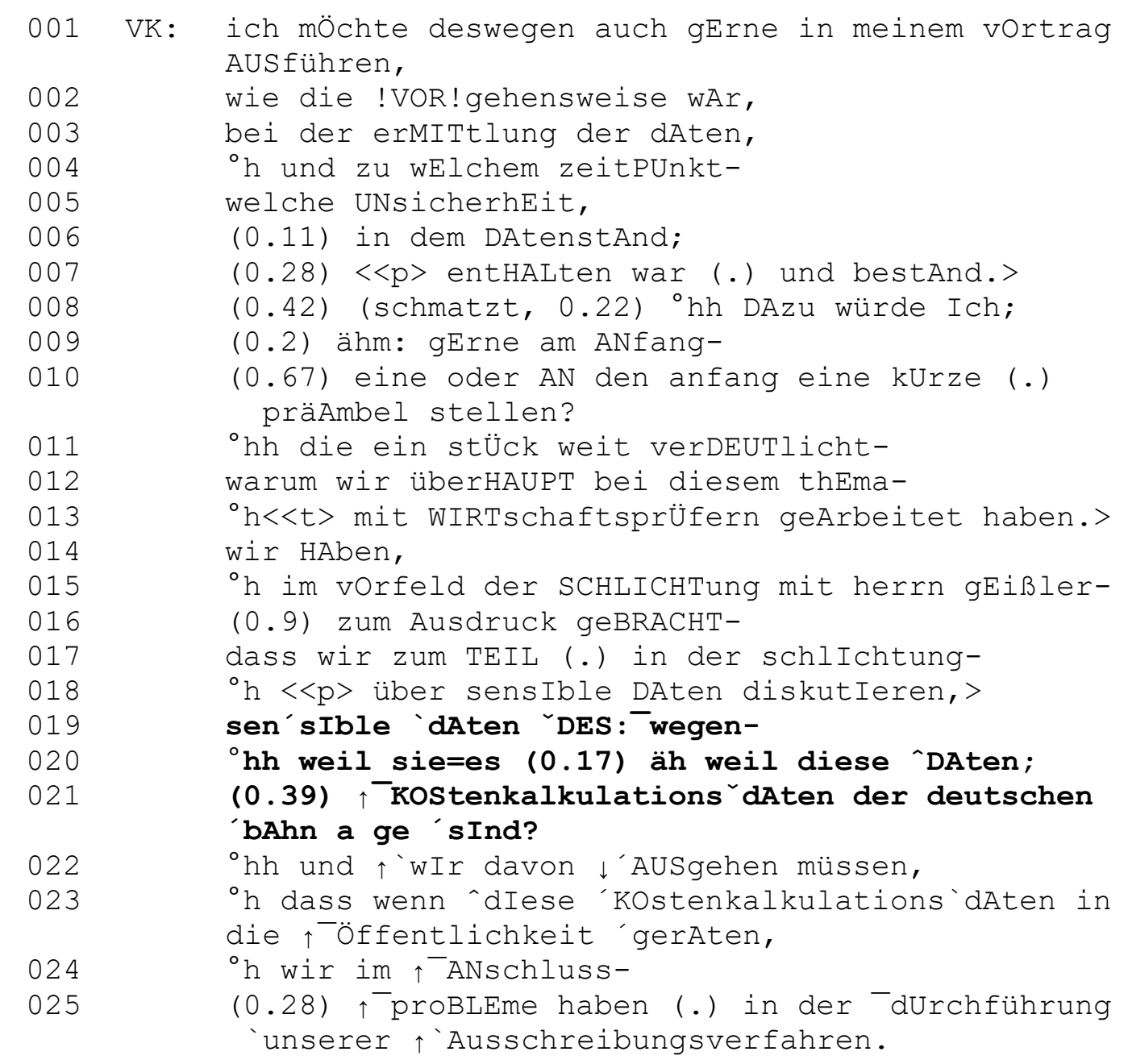

Für diese Verwendung von deswegen (019) liegen zwei syntaktische Lesarten vor, und zwar (i) Nachfeldbesetzung in elliptischem Satz und (ii) Matrixphrase - der Begriff der Matrix erfasst an dieser Stelle die syntaktische Relation der Subordination zwischen einer nicht satzwertigen (Matrix) und einer satzwertigen (Nebensatz) syntaktischen Einheit (vgl. Eisenberg 2013: 47). ${ }^{8}$

\footnotetext{
${ }^{8}$ Eisenberg (2013: 47) führt als Beispiel den zweigliedrigen Satz Karl erwartet, dass Paul ihm schreibt an und geht davon aus, dass das erste Glied Karl erwartet nicht als Hauptsatz bezeichnet werden kann - und zwar aufgrund seiner unvollständigen Argumentstruktur. Deshalb schlägt er für eine breitere Erfassung der Subordination, die über die traditionellen Begriffe des Haupt- und des Nebensatzes hinausgeht, den Rückgriff auf den Begriff der Matrix vor. Der Begriff entstammt der generativen Grammatik, hat sich aber später auch in der Konstruktionsgrammatik etabliert und ist von der Interaktionalen Linguistik übernommen worden. Nennenswerte Beispiele für dessen Anwendung auf die syntaktische Beschreibung des gesprochenen Deutsch sind die in 2.1 genannte [die
} 
Für das Anliegen des vorliegenden Beitrages, nämlich für die Festlegung von Form-FunktionPaaren im Gebrauch der untersuchten Konstruktion erscheint die zweite Lesart m.E. als geeigneter, denn diese Verwendung unterscheidet sich in mehrfacher Hinsicht von den Verwendungen in 3.1. In erster Linie lassen sich Unterschiede auf prosodischer Ebene feststellen. Im Fall der Besetzung des rechten Rands durch deshalb/deswegen wird das erste Glied der Konstruktion auf zwei Intonationsphrasen verteilt - die eine Intonationsphrase trägt die Vorgängerstruktur, die andere den Konnektor - und hat eine bifokale Informationsstruktur. In (3) besteht das erste Glied der Konstruktion aus einer einzelnen Intonationsphrase, dessen Hauptakzent auch hier vom Konnektor getragen wird und die auch hier eine gleichbleibende Tongrenze aufweist:

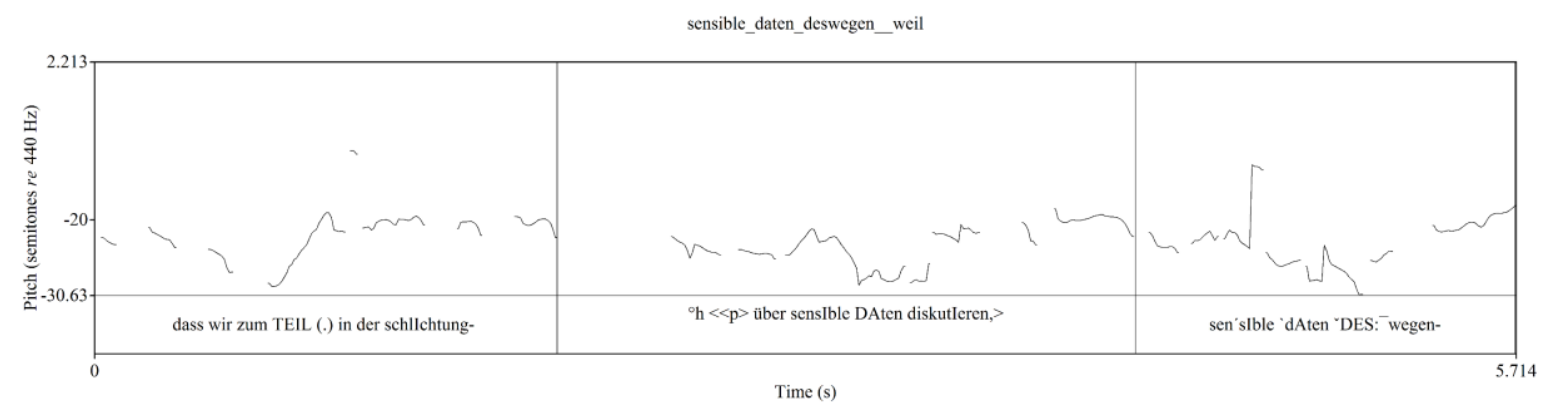

Abbildung 6: Beispiel (3), Tonhöhenverlauf 017-019

Diese Verwendung der Konstruktion hat die gleiche Projektionskraft wie die in 3.1., unterscheidet sich von diesen jedoch hinsichtlich der Relevanzstufung ihrer einzelnen Glieder. Die NP sen'sIble 'dAten ist nur schwach akzentuiert und trägt keine neue Information, sondern nimmt die vorangegangene Informationseinheit wieder auf (über sensIble DAten diskutIeren, 018) und hat insofern relativ präsupponierenden Wert. Die starke Akzentuierung von `DES: ${ }^{-}$wegen weist hingegen auf die Relevanzhochstufung des zweiten Glieds hin, d. h. der syntaktischen Projektion des Konnektors, die eine Proposition mit relativ assertierendem Wert ins Gespräch einbringt. Genauso wie bei der Besetzung des rechten Rands dient diese Matrixphrase zur Überbrückung von einer relevanten Information zur nächsten und somit zum linearen Aufbau des Arguments. Im Vergleich zu Beispielen (1) und (2) liegt jedoch ein wesentlicher Unterschied vor, und zwar in der sequenziellen Einbettung der Konstruktion. Die im letzten Abschnitt angeführten Beispiele für die Besetzung des rechten Rands durch deshalb erscheinen in Sequenzen, in denen die Redner mit relativ hohem Handlungsdruck rechnen müssen. In (1) meldet sich die Rednerin spontan und kurzfristig zu Wort, um einen vorangegangenen Beitrag zu kritisieren, und sie bemüht sich, so viele relevante Informationen wie möglich ins Gespräch einzubringen, um ihre Rederechtübernahme zu rechtfertigen. In (2) ist der Redner mit einem Einwand seitens eines Hörers konfrontiert, d. h. mit einer unerwarteten Gesichtsbedrohung. ${ }^{9}$ Der Redner in (3) markiert seinen Redebeitrag hingegen explizit als Vortrag (001), was m.E. darauf schließen lässt, dass er an dieser Stelle planmäßig zu Wort kommt. Dafür spricht die Tatsache, dass

Sache ist]-Konstruktion in Günthner (2008) und die von Imo (2007) beschriebenen Konstruktionen mit nichteingeleiteten Nebensätzen und abhängigen Hauptsätzen - für die letzteren siehe auch Auer (1998).

${ }^{9}$ Gesichtsbedrohung gilt in der deutschsprachigen Gesprächsforschungsliteratur als Standardübersetzung vom Englischen des Begriffs face threatening act. Der Begriff face entstammt ursprünglich der Soziologie und wurde dann von Brown/Levinson (1987) in der interkulturellen Pragmatik wiedergegeben. 
die Konstruktion in (3) in einer monologischen Sequenz eingebettet ist. Eine ähnliche sequenzielle Einbettung ist auch im folgenden Beispiel zu beobachten:

Beispiel (4): Sprechereignis FOLK_00069; DGS-Kennzeichnung: Schlichtungsgespräch

Redner: Prof. Walter Wittke (Sachverständiger)

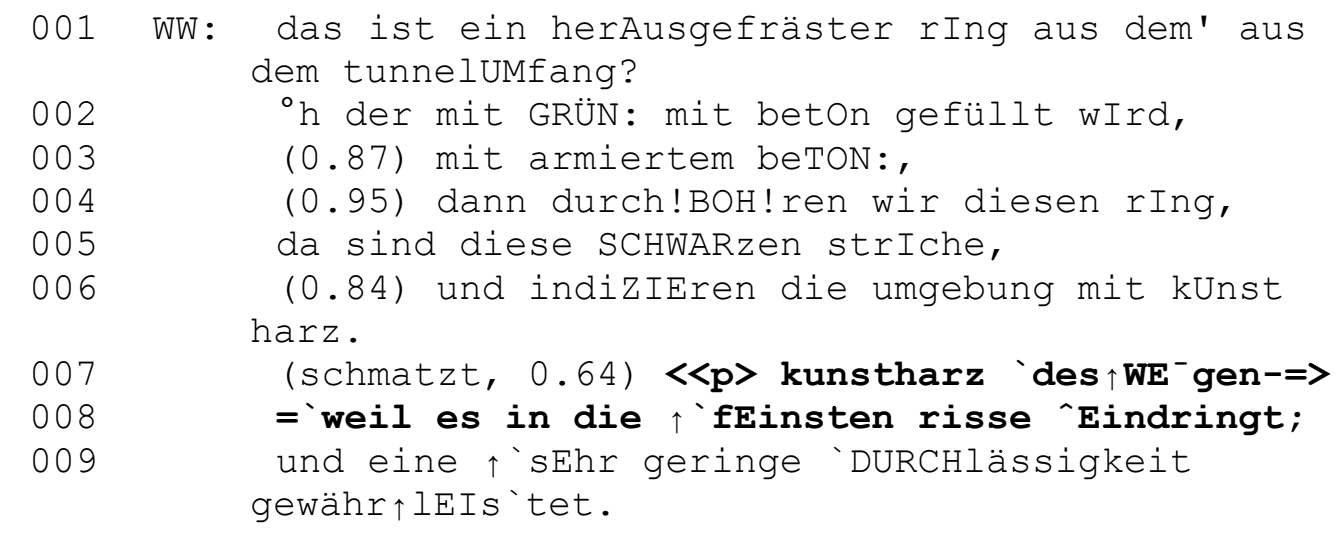

Diese Verwendung der Konstruktion gleicht der in (3) in jeder Hinsicht. Syntaktisch besteht das erste Glied immer noch aus einer [NP+Konnektor]-Phrase. Prosodisch trägt der Konnektor den Hauptakzent seiner Intonationsphrase, an deren Grenze die Intonation gleichbleibt:

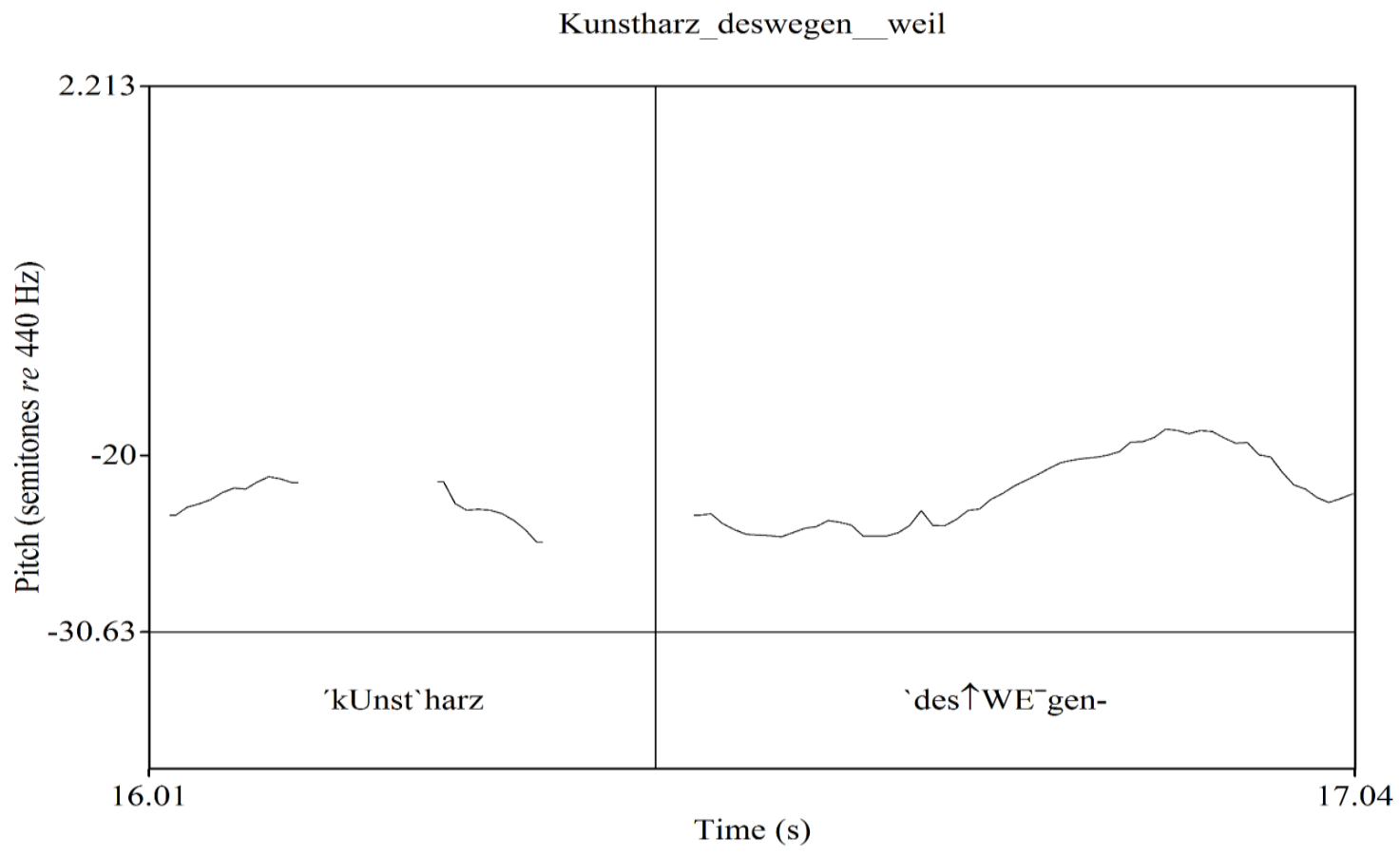

Abbildung 7: Beispiel (4), Tonhöhenverlauf 007

Aus informationsstruktureller Sicht nimmt die topikalisierte NP die vorangegangene Informationseinheit wieder auf, während der Konnektor eine syntaktische Projektion über eine relativ assertierende Proposition auslöst. Beispiele (3) und (4) gleichen sich schließlich auch aus sequenzieller Sicht, denn auch der Redner in (4) ist mit einer monologischen, vortragsmäßigen kommunikativen Aufgabe beschäftigt - er beschreibt nämlich ein technisches Tunnelbauverfahren. 


\section{$4 \quad$ Fazit}

Die Datenanalyse hat gezeigt, wie Projektorkonstruktionen mit deshalb/deswegen als rhetorisches Hilfsmittel zur informationsstrukturellen Gestaltung im Rahmen von argumentaufbauenden kommunikativen Handlungen dienen können. Ihre Projektionsstärke begünstigt die Präsentation von komplexen relevanten Informationsgehalten durch deren Portionierung in kleinere Einheiten. Somit erleichtert der Redner die Bearbeitung der ausgesprochenen Information durch den Hörer und lenkt zugleich seine Aufmerksamkeit auf die noch auszusprechende.

Im Folgenden werden die kennzeichnenden Merkmale der zwei Varianten der [deshalb/deswegen, weil]-Projektorkonstruktion, die im letzten Abschnitt beschrieben worden sind, kurz zusammengefasst.

Der Konnektor deshalb am rechten Rand erscheint in einer von der Vorgängerstruktur prosodisch selbständigen Intonationsphrase mit gleichbleibender Tonkontur. Sowohl der Konnektor als auch seine Vorgängerstruktur tragen einen Hauptakzent, also erhält das erste Glied der Konstruktion eine bifokale Informationsstruktur. Somit wird der propositionale Gehalt des ersten Glieds und der syntaktischen Projektion des Konnektors über das zweite Glied als gleichermaßen relevant markiert. Diese Variante ist in dialogische Sequenzen eingebettet, in denen der jeweilige Redner einen ungeplanten Redebeitrag unter gewissem Handlungsdruck liefert.

In [NP + deswegen]-Matrixphrasen bildet der Konnektor mit seiner Vorgängerstruktur eine einzige Intonationsphrase mit gleichbleibender Tonkontur und trägt den Hauptakzent. Das erste Glied der Konstruktion erhält somit eine monofokale Informationsstruktur. Die topikalisierte NP nimmt eine vorangegangene Informationseinheit wieder auf und ist dementsprechend prosodisch nicht prominent, während der Konnektor eine syntaktische Projektion auslöst, die neue, relevante Information trägt. Diese Variante ist in monologische Sequenzen eingebettet, in denen der Redner eine geplante kommunikative Aufgabe ausführt und einem geringeren Druck ausgesetzt ist.

\section{Literaturverzeichnis}

Altmann, Hans (1981): Formen der „Herausstellung “im Deutschen: Rechtsversetzung, Linksversetzung, freies Thema und verwandte Konstruktionen. Berlin/New York: de Gruyter.

Antonioli, Giorgio (2016): Konnektoren im gesprochenen Deutsch. Eine Untersuchung am Beispiel der kommunikativen Gattung ,, autobiographisches Interview “. Frankfurt a. M.: Lang.

Auer, Peter (1998): „Zwischen Parataxe und Hypotaxe. ,Abhängige Hauptsätze“ im Gesprochenen und Geschriebenen Deutsch“. Zeitschrift für Germanistische Linguistik 26/98: 284-307.

Auer, Peter (2002): „Projection in interaction, projection in grammar“. InList 33/02.

Auer, Peter (2007): „Syntax als Prozess“. In: Hausendorf, Heiko (ed.): Gespräch als Prozess. Tübingen, Narr: 95-124.

Breindl, Eva/Volodina, Anna/Waßner, Ulrich H. (2014): Handbuch der deutschen Konnektoren 2. Berlin/Boston: de Gruyter.

Brown, Penelope/Levinson, Stephen (1987): Politeness. Some universals in language usage. Cambridge: Cambridge University Press.

Eisenberg, Peter (2013): Grundriss der deutschen Grammatik. Bd 2: Der Satz. 4. Aufl. Stuttgart: Metzler. 
Günthner, Susanne (2006): „Was ihn trieb, war vor allem Wanderlust. Pseudocleft-Konstruktionen im Deutschen“. In: Günthner, Susanne/Imo, Wolfgang (eds.): Konstruktionen in der Interaktion. Berlin/New York, de Gruyter: 59-90.

Günthner, Susanne (2008): „Projektorkonstruktionen im Gespräch: Pseudoclefts, die Sache istKonstruktionen und Extrapositionen mit es“. Gesprächsforschung - Online-Zeitschrift zur verbalen Interaktion 9/08: 86-114.

Imo, Wolfgang (2007): Construction Grammar und Gesprochene-Sprache-Forschung. Konstruktionen mit zehn matrixsatzfähigen Verben im gesprochenen Deutsch. Tübingen: Niemeyer.

Imo, Wolfgang (2013): Sprache-in-Interaktion. Analysemethoden und Untersuchungsfelder. Berlin/Boston: de Gruyter.

Nothdurft, Werner (ed.) (1995): Schlichtung. Bd. 1: Streit schlichten. Berlin/New York: de Gruyter. (= Schriften des Instituts für Deutsche Sprache 5.1).

Pasch, Renate et al. (2003): Handbuch der deutschen Konnektoren. Berlin: de Gruyter.

Selting, Margret et al. (2009): „Gesprächsanalytisches Transkriptionssystem 2 (GAT 2)“. Gesprächsforschung - Online-Zeitschrift zur verbalen Interaktion 10/09: 305-402. 


\section{Anhang}

GAT 2-Transkriptkonventionen (nach Selting et al. 2009)

Sequenzielle Struktur/Verlaufsstruktur

$=$ : schneller, unmittelbarer Anschluss neuer Sprecherbeiträge oder Segmente (latching)

Ein- und Ausatmen

${ }^{\circ} \mathrm{h} / \mathrm{h}{ }^{\circ}$ : Ein- bzw. Ausatmen von ca. 0.2-0.5 Sek. Dauer

${ }^{\circ} \mathrm{hh} / \mathrm{hh}{ }^{\circ}$ : Ein- bzw. Ausatmen von ca. 0.5-0.8 Sek. Dauer

${ }^{\circ} \mathrm{hhh} / \mathrm{hhh}{ }^{\circ}$ : Ein- bzw. Ausatmen von ca. 0.8-1.0 Sek. Dauer

\section{$\underline{\text { Pausen }}$}

( . ) : Mikropause, geschätzt, bis ca. 0.2 Sek. Dauer

$(-)$ : kurze geschätzte Pause von ca. 0.2-0.5 Sek. Dauer

$(--)$ : mittlere geschätzte Pause v. ca. 0.5-0.8 Sek. Dauer

$(---)$ : längere geschätzte Pause von ca. 0.8-1.0 Sek. Dauer

$(0.5),(2.0)$ : gemessene Pausen von ca. 0.5 bzw. 2.0 Sek. Dauer (Angabe mit einer Stelle hinter dem Punkt)

Sonstige segmentale Konventionen

und_äh: Verschleifungen innerhalb von Einheiten

äh öh äm: Verzögerungssignale, sog. "gefüllte Pausen"

: :Dehnung, Längung, um ca. 0.2-0.5 Sek.

: : : Dehnung, Längung, um ca. 0.5-0.8 Sek.

: : : : Dehnung, Längung, um ca. 0.8-1.0 Sek.

Tonhöhenbewegung am Ende von Intonationsphrasen

?: hoch steigend

,$:$ mittel steigend

-: gleichbleibend

; : mittel fallend

$\therefore$ : tief fallend

Auffällige Tonhöhensprünge

$\uparrow:$ kleinere Tonhöhensprünge nach oben

$\downarrow$ : kleinere Tonhöhensprünge nach unten

$\uparrow \uparrow:$ größere Tonhöhensprünge nach oben

$\downarrow \downarrow$ : größere Tonhöhensprünge nach unten

Verändertes Tonhöhenregister

$\langle<t\rangle>$ : tiefes Tonhöhenregister

$<<h>>$ : hohes Tonhöhenregister

Akzentuierung

akZENT: Fokusakzent

akzEnt: Nebenakzent 
ak! ZENT ! : extra starker Akzent

Intralineare Notation von Akzenttonhöhenbewegungen

'sO: fallend

'SO: steigend

- SO: gleichbleibend

` $\mathrm{SO}$ : steigend-fallend

sO:fallend-steigend

$\uparrow:$ : kleiner Tonhöhensprung hoch zum Gipfel der Akzentsilbe

$\downarrow^{\prime}$ : kleiner Tonhöhensprung herunter zum Tal der Akzentsilbe

$\uparrow^{-}$SO bzw. $\downarrow^{-}$SO: Tonhöhensprünge zu auffallend höheren bzw. tieferen gleichbleibenden Akzenten

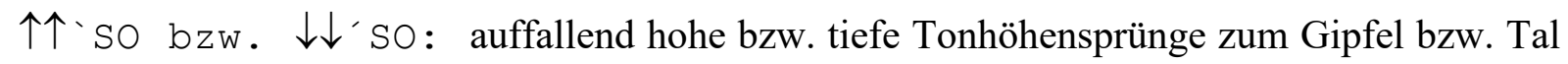
der Akzentsilbe

Lautstärke- und Sprechgeschwindigkeitsveränderungen, mit Extension

$<<f>>$ : forte, laut

$<<f f>>$ :fortissimo, sehr laut

$<<\mathrm{p}\rangle>$ : piano, leise

$<\langle$ pp $\rangle>$ : pianissimo, sehr leise

$<<$ all $>>$ : allegro, schnell

$<<$ len $>>$ : lento, langsam

$<<$ cresc $>>$ : crescendo, lauter werdend

$<<$ dim $>>$ :diminuendo, leiser werdend

$<\langle$ acc $\rangle>$ : accelerando, schneller werdend

$\langle\langle\operatorname{rall}\rangle>$ : rallentando, langsamer werdend 